\section{Relação entre consumo alimentar de vitaminas e minerais, índice de massa corporal e circunferência da cintura: um estudo de base populacional com adultos no Sul do Brasil}

\author{
Relationship between dietary consumption of \\ vitamins and minerals, body mass index, and \\ waist circumference: a population-based study of \\ adults in southern Brazil
}

\section{Relación entre consumo alimentario de vitaminas y minerales, índice de masa corporal y circunferencia de la cintura: un estudio de base poblacional con adultos en el sur de Brasil}

Francieli Cembranel 1

Ana Luiza Curi Hallal 1

David Alejandro González-Chica 2

Eleonora d'Orsi 1

doi: 10.1590/0102-311X00136616

\title{
Resumo
}

O objetivo do estudo foi investigar a associação entre o consumo alimentar inadequado de micronutrientes e indicadores de obesidade geral e abdominal. Análise transversal, da segunda onda do estudo longitudinal EpiFloripa Adultos, incluindo 1.222 indivíduos de 22-63 anos, residentes em Florianópolis, Santa Catarina, Sul do Brasil. Os dados de consumo alimentar foram obtidos mediante aplicação de dois recordatórios de 24 horas, sendo o consumo habitual e as prevalências de inadequação no consumo de cálcio, ferro, zinco, e das vitaminas $A, C, D$ e E estimadas conforme as recomendações do Instituto de Medicina e do Conselho Nacional de Pesquisa dos Estados Unidos. O estado nutricional de obesidade geral foi determinado considerando-se valores do indice de massa corporal (IMC), e a obesidade abdominal, considerandose valores da circunferência da cintura (CC). Para a maioria dos micronutrientes investigados (cálcio, vitaminas $A, C, D$ e E), as médias de consumo mostraram-se aquém dos valores recomendados, com prevalências elevadas de inadequação para toda a amostra. Somente a inadequação no consumo de vitamina $C$ foi maior entre indivíduos obesos (geral ou abdominal). Além disso, associação inversa foi observada entre o menor consumo de cálcio e ferro com valores maiores do IMC e da CC, e entre o menor consumo das vitaminas A e D com valores maiores da CC ( $\beta=-0,92 \mathrm{~cm}$; IC95\%: $-1,76 ;-0,08$ e $\beta=$ -0,69cm; IC95\%: - 1,32; -0,06, respectivamente), principalmente entre adultos jovens. Foi possível identificar a existência de associação inversa entre o consumo alimentar inadequado de micronutrientes e o estado nutricional de obesidade geral e abdominal também em uma amostra de adultos residentes no Sul do Brasil.

Consumo de Alimentos; Micronutrientes; Obesidade

\author{
Correspondência \\ F. Cembranel \\ Programa de Pós-graduação em Saúde Coletiva, Universidade \\ Federal de Santa Catarina. \\ Campus Reitor João David Ferreira Lima s/n, Florianópolis, SC \\ 88040-900, Brasil. \\ francielicembranel@gmail.com
}

1 Programa de Pós-graduação em Saúde Coletiva, Universidade Federal de Santa Catarina, Florianópolis, Brasil

2 Adelaide Medical School, The University of Adelaide,

Adelaide, Australia. 


\section{Introdução}

A obesidade representa o problema nutricional de maior ascensão na população mundial observado nos últimos anos. Com prevalências que oscilam entre 5\% no sudeste da Ásia e 26\% nas Américas, essa doença acomete indivíduos de todas as idades, independentemente de sexo, raça ou classe social 1,2.

Segundo relatório da Organização Mundial da Saúde (OMS), em nível mundial, a prevalência da obesidade começou a aumentar a partir da década de 1980, sendo esse aumento gradual em países de renda alta e com tendência à estabilização nos últimos anos. Por outro lado, em países de renda baixa e média, esse aumento foi tardio e acentuado, cenário no qual se encontra o Brasil, ocupando em nível mundial o terceiro lugar entre os países com maior aumento no número absoluto de pessoas obesas nos últimos trinta anos (20 milhões), atrás apenas da China (42 milhões) e dos Estados Unidos (56 milhões) 1,2 . Nesse contexto, ainda é preciso considerar concomitantemente o aumento da obesidade abdominal. Estima-se, em uma perspectiva global, que pelo menos 30-40\% dos adultos em nível mundial apresentem valores da circunferência da cintura (CC) elevados 3,4,5.

A gravidade desses resultados concentra-se no fato de que a obesidade geral, e sobretudo a abdominal, contribuem para as principais causas de morbimortalidade em todo o mundo, incluindo doenças cardiovasculares, hipertensão, neoplasias e diabetes mellitus tipo 2. Além do impacto sobre a qualidade de vida, a obesidade prejudica também o desenvolvimento socioeconômico dos países, devido aos custos relacionados com o tratamento das complicações, hospitalizações e pelos anos potenciais de trabalho perdidos 6 .

A má qualidade da dieta tem sido apontada como uma das principais causas desse cenário. O que se observa na atualidade é um aumento do consumo calórico total advindo de um maior consumo de carboidratos simples e gorduras saturadas, em detrimento do consumo de alimentos fontes de micronutrientes essenciais 7 . Como resultados, pesquisas realizadas tanto em países de renda alta 8,9,10 quanto média 11,12 têm encontrado associações entre o consumo alimentar insuficiente de micronutrientes (cálcio, ferro, zinco, vitaminas A, C, D e E) e a condição de obesidade geral e/ou abdominal.

No Brasil, contudo, é preciso considerar que, até o momento, a associação entre o consumo insuficiente de micronutrientes e a obesidade não tem sido suficientemente explorada. Isso ocorre porque o único trabalho de base populacional realizado no país 13 teve como objetivo principal comparar as prevalências de inadequação no consumo alimentar de algumas vitaminas e minerais (zinco, vitaminas $\mathrm{A}, \mathrm{C}$ e E) entre indivíduos obesos e não obesos. Entretanto, não houve avanços em termos de análises de associação entre as deficiências no consumo de micronutrientes e a obesidade geral e abdominal, o que reforça a importância desse tipo de investigação no país, uma vez que no Brasil 14, assim como em outros países de renda média 15 , a elevada prevalência de obesidade coexiste com graves deficiências no consumo de micronutrientes (geralmente com oscilação de 40-100\% para o cálcio e as vitaminas A, C, D e E).

Frente ao exposto, o objetivo do presente estudo foi investigar a associação entre o consumo alimentar inadequado de micronutrientes (cálcio, ferro, zinco, vitaminas A, C, D e E) e indicadores de obesidade geral e abdominal entre adultos residentes em uma capital do Sul do Brasil.

\section{Métodos}

\section{Delineamento e local do estudo}

Trata-se de uma análise transversal cuja amostra da investigação foi composta por indivíduos com idade entre 22 e 63 anos, que participaram da segunda onda do estudo longitudinal de base populacional, EpiFloripa Adultos.

O EpiFloripa Adultos iniciou-se em 2009 (linha de base) com o objetivo de examinar condições de vida e saúde geral de uma amostra representativa de adultos residentes em Florianópolis, capital do Estado de Santa Catarina, Região Sul do Brasil. O levantamento inicial incluiu adultos de 20 a 59 anos de idade, residentes na área urbana de Florianópolis, grupo populacional que representava aproximadamente $60 \%$ da população total do município naquele ano (408.161 habitantes) 16. Em 2012, visando 
a dar continuidade à investigação, foi realizada a segunda onda do EpiFloripa Adultos, da qual foram obtidos os dados para o presente estudo.

\section{Amostra e amostragem}

Para o cálculo do tamanho da amostra do EpiFloripa Adultos na linha de base, foram considerados como parâmetros uma população de referência de 249.530 adultos ( 20 a 59 anos), prevalência esperada para desfechos desconhecidos de $50 \%$, erro amostral de 3,5 pontos percentuais, intervalo de $95 \%$ de confiança (IC95\%), efeito de delineamento de 2 (devido a amostragem por conglomerados) e percentual de perdas igual a $10 \%$. Tendo em vista o ajuste para possíveis fatores de confusão, aumentou-se em mais $15 \%$ o tamanho da amostra, sendo esta estimada em 2.016 adultos.

A amostragem foi realizada por conglomerados em dois estágios. No primeiro estágio, foram selecionados sistematicamente 63/420 setores censitários, conforme a renda média mensal do chefe da família (R\$ 192,80 a 13.209,50), e no segundo estágio, os domicílios (1.134/16.755). Todos os residentes de 20 a 59 anos nos domicílios sorteados foram considerados elegíveis para participar do estudo, resultando em uma amostra final de 1.720 adultos efetivamente entrevistados. Detalhes adicionais sobre aspectos metodológicos do EpiFloripa Adultos 2009 encontram-se disponíveis em publicação prévia 16 .

No ano de 2012, todos os entrevistados da linha de base foram convidados a participar de nova entrevista. Os indivíduos que se negaram a participar da nova entrevista foram considerados recusas, enquanto os participantes que não foram encontrados após quatro tentativas (tanto presenciais quanto por telefone) foram considerados perdas. A amostra final resultou em 1.222 adultos de 22 a 63 anos entrevistados em 2012 (Figura 1).

\section{Coleta de dados}

Tanto em 2009 quanto em 2012, as entrevistas foram realizadas por equipe treinada, sendo utilizado um ara registro dos dados. A exceção foram os dados de consumo alimentar, cujo registro em 2012 foi feito em instrumento impresso. Os questionários utilizados em ambas as ondas foram pré-testados. Realizou-se ainda controle de qualidade dos dados coletados, com repetição de perguntas-chave em amostra aleatória de 15\% dos respondentes em 2009 e 10\% em 2012 (valores de kappa entre 0,6 e 1,0).

\section{Desfechos}

Foram consideradas como variáveis dependentes a obesidade geral e a obesidade abdominal.

A obesidade geral foi determinada a partir do cálculo do índice de massa corporal (IMC) ${ }^{17}$. Para tanto, o peso corporal foi aferido por meio de balança digital portátil (modelo HCM 5110 M; GAMA Italy Professional, San Pietro in Casale, Itália) com capacidade de $150 \mathrm{~kg}$, sensibilidade de $100 \mathrm{~g}$ e calibrada antes do trabalho de campo. A altura foi mensurada utilizando-se um estadiômetro portátil desenvolvido especificamente para o estudo, com capacidade máxima de $200 \mathrm{~cm}$ e resolução de $1 \mathrm{~mm}$. Todos os entrevistados foram pesados e medidos seguindo procedimento padrão descrito na literatura 18 . No presente estudo, o IMC foi mensurado de forma contínua $\left(\mathrm{kg} / \mathrm{m}^{2}\right)$ e também como variável categórica dicotômica para mensuração da obesidade geral (IMC $<30 \mathrm{~kg} / \mathrm{m}^{2}$ : sem obesidade geral; IMC $\geq 30 \mathrm{~kg} / \mathrm{m}^{2}$ : com obesidade geral) 17 .

A obesidade abdominal, por sua vez, foi determinada por meio da medida da CC. Para a aferição da CC, foi utilizada uma fita antropométrica inelástica da marca Sanny (São Bernardo do Campo, Brasil), com $160 \mathrm{~cm}$ de comprimento e resolução de $1 \mathrm{~mm}$. A medida da CC foi aferida na região mais estreita do tronco ou, quando não aparente, no ponto médio entre a última costela e a borda superior da crista ilíaca 18. A CC foi mensurada de forma contínua $(\mathrm{cm})$ e também como variável categórica dicotômica para mensuração da obesidade abdominal $(C C<88 \mathrm{~cm}$ em mulheres e $<102 \mathrm{~cm}$ em homens: sem obesidade abdominal; $\mathrm{CC} \geq 88 \mathrm{~cm}$ em mulheres e $\geq 102 \mathrm{~cm}$ em homens: com obesidade abdominal) 19 . 


\section{Exposições principais}

Foi considerado como variável de exposição o consumo alimentar de cálcio $(\mathrm{mg})$, ferro $(\mathrm{mg})$, zinco $(\mathrm{mg})$, vitamina $\mathrm{A}(\mu \mathrm{gRE})$, vitamina $\mathrm{C}(\mathrm{mg})$, vitamina $\mathrm{D}(\mu \mathrm{g})$ e vitamina $\mathrm{E}(\mathrm{mg})$.

Os dados referentes ao consumo alimentar desses micronutrientes foram obtidos pela aplicação presencial de um primeiro recordatório alimentar de 24 horas (R24h) no total da amostra do EpiFloripa Adultos 2012, e de um segundo R24h aplicado por telefone em uma subamostra de 40\% dos participantes 20,21, os quais foram selecionados sistematicamente dentre aqueles que responderam o primeiro R24h no domicílio (um a cada três respondentes). A aplicação do segundo R24h ocorreu em um período de até 15 dias após a aplicação do primeiro, e todos os R24h foram aplicados conforme o Multiple Pass Method 22. As duas coletas foram estruturadas para se obter informações sobre o consumo alimentar de um dia de semana e outro do final de semana, metodologia que possibilitou a correção da distribuição da estimativa de nutrientes 20,21.

As informações obtidas dos dois R24h foram inseridas no software Nutrition Data System for Research (NDSR; Universidade de Minnesota, Mineápolis, Estados Unidos), seguindo, para tanto, padronização recomendada por Fisberg \& Marchioni 23 para inquéritos brasileiros. Nessa etapa, as medidas caseiras dos alimentos foram convertidas em gramas, mililitros ou litros mediante o auxílio de tabela brasileira de medidas caseiras 24 . Considerando ainda que o NDSR apresenta como base de dados principal a tabela do Departamento de Agricultura dos Estados Unidos (USDA), os alimentos típicos brasileiros que não constavam no software tiveram seus valores nutricionais inseridos de acordo com dados nacionais 25. Desse modo, foram obtidas do NDSR informações nutricionais referentes à ingestão de energia e de micronutrientes para cada participante. Nessas estimativas, não foi incluído o consumo de suplementos vitamínicos e/ou medicamentos.

Seguindo as recomendações do Instituto de Medicina (IoM) e do Conselho Nacional de Pesquisa, dos Estados Unidos, todas as variáveis dietéticas foram ajustadas pela variabilidade intraindividual e interindividual, por meio da fórmula Ingesta Ajustada $=[$ (média do sujeito - média do grupo $) \mathrm{x}$ (desvio padrão interpessoal/desvio padrão observado)] + média grupo 20,26,27,28. Adicionalmente, todas essas variáveis ainda passaram por um segundo processo de ajuste pela ingestão de energia pela dieta, seguindo o método de ajuste por resíduos proposto por Willett et al. 20. Todas as variáveis geradas foram analisadas de forma contínua, considerando as recomendações do IoM para cada micronutriente 29,30,31.

\section{Covariáveis}

Com base na literatura 7,9,11, foram considerados como possíveis fatores de confusão as variáveis sexo (feminino, masculino), idade (20-29, 30-49, 50-63 anos), cor da pele (branca, preta/parda), estado civil (solteiro, casado/morando com companheiro, separado/divorciado/viúvo), escolaridade (0-4, 5-8, 9-11, $\geq 12$ anos de estudo) e renda familiar mensal per capita (1o terço: inferior $\leq \mathrm{R} \$ 666,70$; 2o terço: intermediário $>\mathrm{R} \$ 666,70 \mathrm{a} \leq \mathrm{R} \$ 2.050,00$; 3o terço: superior $>\mathrm{R} \$ 2.050,00$; salário mínimo vigente em $2012=R \$ 622,00)$.

\section{Análise dos dados}

A análise dos dados foi conduzida no software Stata versão 13.0 (StataCorp LP, College Station, Estados Unidos). Todas as análises foram realizadas utilizando-se pesos amostrais, considerando o efeito do desenho amostral na linha de base e a probabilidade de localização dos participantes na segunda onda do estudo, de acordo com cada setor censitário (cluster). Esses pesos amostrais ainda foram recalculados para a população estimada de Florianópolis em 2012, buscando, assim, manter a representatividade da amostra em relação à população do município naquele ano.

Para a descrição das variáveis categóricas, os dados foram apresentados em frequências absolutas e relativas, com seus respectivos IC $95 \%$, utilizando-se o teste de qui-quadrado com correção de Rao-Scott. Para a descrição das variáveis contínuas, os dados foram expressos em médias com seus respectivos desvios padrão, sendo o teste t utilizado nessas análises. 
Para avaliar a associação entre os desfechos (IMC e CC contínuos) e cada variável de exposição (micronutrientes), utilizou-se a regressão linear, tanto na análise bruta quanto na análise ajustada, e os dados foram apresentados na forma de coeficientes de regressão ( $\beta$ ) com seus respectivos IC95\%. O valor de significância estatística adotado foi de $\mathrm{p} \leq 0,05$. Coeficientes de determinação ( $\left.\mathrm{R}^{2}\right)$ como medida de explicação do modelo também foram calculados. Os possíveis fatores de confusão foram incluídos de forma conjunta na análise ajustada, independentemente do nível de significância estatística.

Testes de heterogeneidade nas associações, conforme a idade e o sexo, também foram realizados, sendo considerados como indicativos de interação valores de $p \leq 0,10$. Os resultados da modificação de efeito da associação entre as variáveis de exposição e o estado nutricional conforme a idade foram apresentados de forma gráfica. Adicionalmente, devido à natureza transversal das análises, a presença de obesidade geral e abdominal na linha de base também foi testada como possível fonte de heterogeneidade dos resultados encontrados por este estudo 32.

Prevalências de inadequação no consumo de cada micronutriente segundo o estado nutricional da amostra também foram estimadas, utilizando-se como ponto de corte os valores da estimated average requirement (EAR) 29,30,31. A prevalência de inadequação no consumo de cada micronutriente foi determinada considerando a proporção de indivíduos com o consumo abaixo do valor da EAR, dada pela fórmula Escore-Z = (média ajustada $-\mathrm{EAR}) /$ desvio padrão da distribuição ajustada 26 .

\section{Aspectos éticos}

O EpiFloripa Adultos foi aprovado em ambas as ondas pelo Comitê de Ética em Pesquisa com Seres Humanos da Universidade Federal de Santa Catarina (processos 351/2008 e 1.772/2011), e todos os participantes assinaram um Termo de Consentimento Livre e Esclarecido.

\section{Resultados}

Na linha de base do EpiFloripa Adultos, foram entrevistados 1.720 indivíduos (Figura 1). Desses indivíduos, foram localizados e entrevistados novamente 1.222 adultos em 2012 (71\% dos entrevistados na linha de base). As taxas de resposta para o primeiro e segundo R24h foram de 98,7\% e $98,9 \%$, respectivamente.

Nas duas ondas, houve maior proporção de mulheres e de pessoas casadas/morando com companheiro, e não houve diferenças nos percentuais de acompanhamento conforme a cor da pele e a escolaridade. Todavia, houve maior proporção de perdas entre o sexo masculino, na faixa etária de 20 a 29 anos, de solteiros e de participantes no terço inferior de renda. Mesmo assim, $60 \%$ ou mais dos indivíduos nessas categorias foram localizados (Tabela 1). Em 2012, a média de idade da amostra foi de 41,9 anos ( $\pm 11,3$ ); a mediana de renda familiar per capita, de R $\$ 1.125,00$ (p25-p75 R \$ 666,70-R \$ 2.050,00); e a mediana de escolaridade, de 11 anos de estudo (p25-p75: 9-15) (dados não apresentados em tabelas). A obesidade geral foi diagnosticada em 20,2\% (IC95\%: 15,0; 25,8) da população do estudo, enquanto a obesidade abdominal esteve presente em 28,5\% (IC95\%: 23,8; 33,5) da amostra.

Quanto ao consumo alimentar de micronutrientes (Tabela 2), com exceção do consumo de ferro e zinco, para todos os demais micronutrientes, as médias de consumo ficaram abaixo dos valores da EAR, fato que repercutiu em prevalências elevadas de inadequação para a maioria dos micronutrientes investigados. Em particular, a prevalência de inadequação da vitamina $C$ foi maior entre indivíduos com obesidade, seja esta geral ou abdominal (valor de $\mathrm{p}<0,05 \mathrm{em}$ ambos os casos). Além disso, merece destaque o consumo de vitamina $\mathrm{D}$, o que se mostrou inadequado para a totalidade da amostra, independentemente do estado nutricional.

$\mathrm{Na}$ Tabela 3, são apresentadas as análises brutas e ajustadas da associação entre as variáveis de exposição (micronutrientes) e os dois indicadores de estado nutricional. $\mathrm{Na}$ análise bruta, observou-se uma associação inversa entre o IMC e todos os micronutrientes analisados, mas somente o consumo de cálcio, ferro e vitamina A apresentaram resultado estatisticamente significante. Na análise ajustada, a direção das associações manteve-se a mesma, mas com resultados estatisticamente significantes para o cálcio ( $\beta=-0,40 \mathrm{~kg} / \mathrm{m}^{2} ;$ IC95\%: $\left.-0,69 ;-0,12\right)$ e o ferro ( $\beta=-0,33 \mathrm{~kg} / \mathrm{m}^{2} ; \mathrm{IC} 95 \%$ : $\left.-0,57 ;-0,08\right)$. No caso da CC, o consumo de cálcio e de ferro também se mostraram associados de forma negativa 


\section{Figura 1}

Fluxograma de acompanhamento da amostra do estudo EpiFloripa Adultos, ondas 2009 (linha de base) e 2012. Florianópolis, Santa Catarina, Brasil, 2012.

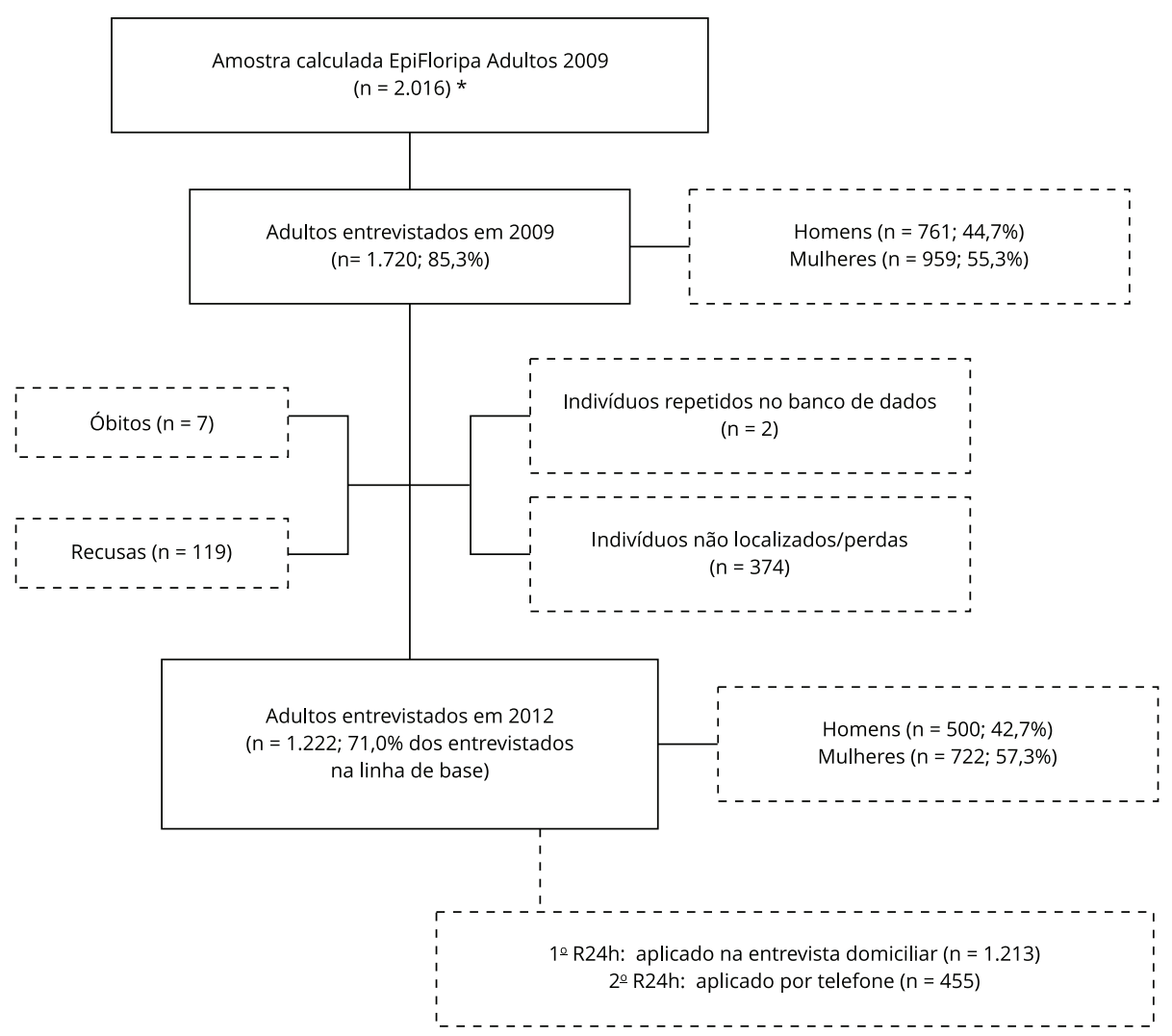

R24h: recordatório alimentar de 24 horas.

* O tamanho estimado da amostra do EpiFloripa Adultos em 2009 considerou a inclusão de 32 indivíduos em cada um dos 63 setores censitários de Florianópolis (18 domicílios sorteados em cada setor, com uma média de 1,7 adultos por domicílio).

com esse desfecho nas análises ajustadas, com R2 de 23,4\% e de 22,7\%, respectivamente. Significância estatística também foi observada nas análises com as vitaminas A ( $\beta=-0,92 \mathrm{~cm}$; IC95\%: -1,76; -0,08) e D ( $\beta=-0,69 \mathrm{~cm}$; IC95\%: -1,32; -0,06). A incorporação de termos quadráticos não modificou o R2 explicado pelo modelo.

No que se refere à modificação de efeito pela idade, foram observados resultados consistentes de heterogeneidade nas associações entre o consumo alimentar de cálcio, vitamina A e vitamina D com os dois indicadores de estado nutricional (Figura 2). Entre os mais jovens (20-29 anos), o menor consumo dos três micronutrientes esteve associado a maiores valores do IMC e da CC. Já entre aqueles com idade entre 50 a 63 anos, os valores $\beta$ mostraram, de forma geral, uma relação direta entre o consumo dos micronutrientes e os valores do IMC e da CC, embora os coeficientes não fossem estatisticamente significantes. No grupo com idade intermediária (30-49 anos), de forma geral, a relação foi nula. A idade também mostrou-se um importante modificador de efeito nas associações entre o consumo de ferro e zinco com a CC (Figura 3), mas não com o IMC (valor de p de interação de 0,359 para o ferro e de 0,227 para o zinco). Contudo, ressalta-se que, para esses micronutrientes, a heterogeneidade dos efeitos foi menor que aquela observada para o cálcio e as vitaminas A e D. 


\section{Tabela 1}

Comparação das características demográficas, socioeconômicas e de estado nutricional da população do estudo EpiFloripa Adultos, na linha de base em 2009 e na segunda onda em 2012. Florianópolis, Santa Catarina, Brasil, 2012.

\begin{tabular}{|c|c|c|c|c|}
\hline Variáveis & $\begin{array}{l}\text { Amostra na linha de base } \\
\qquad(2009)[N=1.720]\end{array}$ & $\begin{array}{l}\text { Amostra da 2a onda } \\
(2012)[N=1.222]\end{array}$ & $\begin{array}{l}\text { Percentual da coorte } \\
\text { entrevistada em } \\
2012 \text { em relação aos } \\
\text { entrevistados } \\
\text { em } 2009 \\
\% * *\end{array}$ & Valor de $p$ * \\
\hline \multicolumn{5}{|l|}{ Sexo } \\
\hline Masculino & $761(44,2)$ & $522(48,2)$ & 68,6 & 0,046 \\
\hline Feminino & $959(55,8)$ & $700(51,8)$ & 73,0 & \\
\hline \multicolumn{5}{|l|}{ Idade (anos) } \\
\hline $20-29$ & $540(31,4)$ & $328(26,8)$ & 60,7 & $<0,001$ \\
\hline $30-39$ & $392(22,8)$ & $277(22,7)$ & 70,7 & \\
\hline $40-49$ & $438(25,5)$ & $345(28,2)$ & 78,8 & \\
\hline $50-63$ & $350(20,3)$ & $272(22,3)$ & 77,7 & \\
\hline \multicolumn{5}{|l|}{ Cor da pele } \\
\hline Branca & $1.534(89,6)$ & $1.092(89,7)$ & 71,2 & 0,911 \\
\hline Preta/Parda & $178(10,4)$ & $126(10,3)$ & 70,8 & \\
\hline \multicolumn{5}{|l|}{ Estado civil } \\
\hline Casado/Morando com companheiro & $1.043(60,6)$ & $779(63,8)$ & 74,7 & $<0,001$ \\
\hline Solteiro/Divorciado/Viúvo & $677(39,4)$ & $443(36,2)$ & 65,4 & \\
\hline \multicolumn{5}{|l|}{ Escolaridade (anos de estudo) } \\
\hline $0-4$ & $158(9,2)$ & $108(8,9)$ & 68,4 & 0,217 \\
\hline $5-8$ & $253(14,7)$ & $174(14,3)$ & 68,8 & \\
\hline $9-11$ & $568(33,1)$ & $394(32,3)$ & 69,4 & \\
\hline 12 ou mais & $737(43,0)$ & $543(44,5)$ & 73,7 & \\
\hline \multicolumn{5}{|l|}{ Renda familiar per capita } \\
\hline 1 o terço (inferior) & $564(33,5)$ & $382(31,7)$ & 67,7 & 0,040 \\
\hline 2o terço (intermediário) & $562(33,4)$ & $409(33,9)$ & 72,8 & \\
\hline 3o terço (superior) & $559(33,1)$ & $415(34,4)$ & 74,2 & \\
\hline \multicolumn{5}{|l|}{ Obesidade geral } \\
\hline Não (IMC < 30kg/m²) & $1.406(83,8)$ & $910(79,8)$ & 64,7 & 0,026 \\
\hline $\operatorname{Sim}\left(\mathrm{IMC} \geq 30 \mathrm{~kg} / \mathrm{m}^{2}\right)$ & $271(16,2)$ & $230(20,2)$ & 84,9 & \\
\hline \multicolumn{5}{|l|}{ Obesidade abdominal $* \star \star$} \\
\hline Não & $1.385(80,5)$ & $874(71,5)$ & 63,1 & 0,107 \\
\hline Sim & $335(19,5)$ & $348(28,5)$ & 100,0 & \\
\hline
\end{tabular}

IMC: índice de massa corporal.

* Valor de p do teste de qui-quadrado, comparando entrevistados em 2012 em relação aos não entrevistados;

** Frequência relativa;

*** Obesidade abdominal: não (quando a medida da circunferência da cintura - CC $<88 \mathrm{~cm}$ em mulheres e $<102 \mathrm{~cm}$ em homens; sim (quando a medida da CC $\geq 88 \mathrm{~cm}$ em mulheres $\mathrm{e} \geq 102 \mathrm{~cm}$ em homens) 19 .

Somente no caso das vitaminas $\mathrm{C}$ e $\mathrm{E}$ a idade não foi modificadora de efeito para nenhum dos dois desfechos investigados (valores de $\mathrm{p}$ de interação $>0,10$ ).

Por fim, os testes de heterogeneidade conduzidos com a variável sexo mostraram que essa variável não foi modificadora de efeito nas análises realizadas, assim como também não foi observada qualquer evidência de modificação de efeito nas associações encontradas em 2012 relacionada com a presença de obesidade geral e abdominal na linha de base (valor de $\mathrm{p}$ de interação $>0,10 \mathrm{em}$ todos os casos; resultados não apresentados em tabelas). 


\section{Tabela 2}

Consumo alimentar de micronutrientes e prevalências de inadequação, segundo estado nutricional da amostra. Estudo EpiFloripa Adultos 2012 ( $\mathrm{n}=1.051)$ *, Florianópolis, Santa Catarina, Brasil, 2012.

\begin{tabular}{|c|c|c|c|c|c|c|}
\hline \multirow[t]{3}{*}{ Variáveis } & \multicolumn{3}{|c|}{ Obesidade geral ** } & \multicolumn{3}{|c|}{ Obesidade abdominal *** } \\
\hline & Não & Sim & Valor de $\mathbf{p} \#$ & Não & Sim & Valor de $\mathbf{p} \#$ \\
\hline & Média (DP) & Média (DP) & & Média (DP) & Média (DP) & \\
\hline Cálcio (mg) & $777,6(218,3)$ & $732,1(206,2)$ & 0,128 & $777,7(217,9)$ & $738,2(210,0)$ & 0,356 \\
\hline Ferro (mg) & $11,6(1,0)$ & $11,5(0,8)$ & 0,038 & $11,6(1,0)$ & $11,5(0,88)$ & 0,068 \\
\hline Zinco (mg) & $10,5(2,5)$ & $10,3(1,9)$ & 0,592 & $10,5(2,6)$ & $10,3(1,9)$ & 0,972 \\
\hline Vitamina A ( $\mu g R E)$ & $296,2(94,1)$ & $280,5(98,2)$ & 0,228 & $294,0(91,5)$ & $290,4(106,8)$ & 0,835 \\
\hline Vitamina C (mg) & $57,1(40,8)$ & $51,2(37,5)$ & 0,048 & $56,1(40,1)$ & $55,8(41,1)$ & 0,406 \\
\hline Vitamina D $(\mu g)$ & $3,5(1,5)$ & $3,4(1,4)$ & 0,383 & $3,5(1,5)$ & $3,4(1,4)$ & 0,544 \\
\hline Vitamina E (mg) & $5,8(2,4)$ & $5,8(2,4)$ & 0,247 & $5,8(2,4)$ & $5,9(2,4)$ & 0,467 \\
\hline Prevalências de inadequação \#\# & $\%$ & $\%$ & Valor de p \#\#\# & $\%$ & $\%$ & Valor de $p \# \# \#$ \\
\hline Cálcio (mg) & 59,7 & 70,8 & 0,386 & 58,7 & 71,9 & 0,419 \\
\hline Ferro (mg) & 0,0 & 0,0 & 1,000 & 0,0 & 0,0 & 1,000 \\
\hline Zinco (mg) & 18,9 & 15,4 & 0,928 & 20,2 & 11,5 & 0,471 \\
\hline Vitamina A ( $\mu g R E)$ & 99,0 & 99,5 & 0,060 & 98,3 & 99,3 & 0,924 \\
\hline Vitamina C (mg) & 58,0 & 65,0 & 0,044 & 58,3 & 59,6 & 0,049 \\
\hline Vitamina D $(\mu g)$ & 100,0 & 100,0 & 0,113 & 100,0 & 100,0 & 0,189 \\
\hline Vitamina E (mg) & 99,6 & 99,2 & 0,311 & 99,6 & 99,3 & 0,297 \\
\hline
\end{tabular}

DP: desvio padrão.

Nota: os valores apresentados na tabela referem-se a uma prevalência ponderada dos valores encontrados em homens e mulheres para cada faixa etária e para cada indicador de estado nutricional 29,30,31.

* A amostra analítica foi constituída por um n=1.051, uma vez que foram incluídos nas análises apenas os registros com dados completos para todas as variáveis;

** Obesidade geral: não (quando o valor do índice de massa corporal - IMC < 30kg/m2; sim (quando o valor do IMC $\geq 30 \mathrm{~kg} / \mathrm{m} 2$ ) 17;

*** Obesidade abdominal: não (quando a medida da circunferência da cintura - CC $<88 \mathrm{~cm}$ em mulheres e $<102 \mathrm{~cm}$ em homens); sim (quando a

medida da CC $\geq 88 \mathrm{~cm}$ em mulheres e $\geq 102 \mathrm{~cm}$ em homens) 19 ;

\# Valor de $p$ do teste t;

\#\# A prevalência de inadequação no consumo de todos os micronutrientes foi obtida empregando-se a fórmula do escore-Z e os pontos de corte da Estimated Average Requirement (EAR): cálcio: $800 \mathrm{mg} / \mathrm{dia}$ homens, $800 \mathrm{mg} / \mathrm{dia}$ mulheres de $20-50$ anos, $1.000 \mathrm{mg} / \mathrm{dia} \mathrm{mulheres} \mathrm{com} \mathrm{+50} \mathrm{anos;} \mathrm{ferro:} 6 \mathrm{mg} /$ dia homens, 8,1 mg/dia mulheres de $20-50$ anos, 5mg/dia mulheres com +50 anos; zinco: 9,4mg/dia homens, 6,8mg/dia mulheres; vitamina a: $625 \mu$ g/dia homens, 500 $\mu$ g/dia mulheres; vitamina c: $75 \mathrm{mg} /$ dia homens, 60mg/dia mulheres; vitamina d: $10 \mu \mathrm{g} / \mathrm{dia}$ homens, $10 \mu \mathrm{g} / \mathrm{dia}$ mulheres; vitamina e: $12 \mathrm{mg} /$ dia homens, $12 \mathrm{mg}$ /dia mulheres;

\#\#\# Valor de p do teste de qui-quadrado.

\section{Discussão}

Com base nos resultados encontrados, três conclusões principais podem ser mencionadas. Primeiro, para a maioria dos micronutrientes investigados (cálcio, vitaminas A, C, D e E), as médias de consumo mostraram-se aquém dos valores recomendados, repercutindo em prevalências elevadas de inadequação para toda a amostra. Em particular, o consumo inadequado de vitamina $\mathrm{C}$ foi maior entre indivíduos com obesidade (geral e abdominal). Segundo, com base nos resultados das análises ajustadas de associação, neste estudo, foi possível também confirmar as evidências da literatura, ou seja, a existência de associação inversa entre o consumo alimentar insuficiente de cálcio e ferro e o IMC elevado, e de associação inversa entre o consumo alimentar insuficiente de cálcio, ferro e das vitaminas A e D e a CC elevada. Terceiro, a idade mostrou-se um importante modificador de efeito nas associações entre o consumo alimentar de micronutrientes e os dois indicadores de estado nutricional. Entre os indivíduos de 20 a 29 anos, observou-se que o menor consumo de cálcio, vitamina 


\section{Tabela 3}

Análise bruta e ajustada da associação entre o consumo alimentar de micronutrientes e indicadores de obesidade geral e abdominal. Estudo EpiFloripa Adultos 2012 ( $\mathrm{n}=1.051)$ *, Florianópolis, Santa Catarina, Brasil, 2012.

\begin{tabular}{|c|c|c|c|c|c|c|}
\hline \multirow[t]{2}{*}{ Variáveis } & \multicolumn{3}{|c|}{ Regressão linear bruta } & \multicolumn{3}{|c|}{ Regressão linear ajustada ** } \\
\hline & $\beta$ & $R^{2}(I C 95 \%)$ & Valor de $p * * *$ & $\beta$ & $R^{2}($ IC95\%) & Valor de $p * * *$ \\
\hline \multicolumn{7}{|l|}{ IMC (kg/m²) } \\
\hline Cálcio (mg) & $-0,54$ & $1,2(-0,85 ;-0,24)$ & 0,001 & $-0,40$ & $8,5(-0,69 ;-0,12)$ & 0,006 \\
\hline Ferro (mg) & $-0,33$ & $0,5(-0,62 ;-0,04)$ & 0,027 & $-0,33$ & $8,3(-0,57 ;-0,08)$ & 0,011 \\
\hline Zinco (mg) & $-0,06$ & $0,0(-0,43 ; 0,31)$ & 0,754 & $-0,12$ & $7,9(-0,39 ; 0,15)$ & 0,385 \\
\hline Vitamina A ( $\mu g R E)$ & $-0,35$ & $0,5(-0,68 ;-0,02)$ & 0,039 & $-0,33$ & $8,3(-0,67 ; 0,02)$ & 0,062 \\
\hline Vitamina C (mg) & $-0,15$ & $0,1(-0,55 ; 0,24)$ & 0,440 & $-0,28$ & $8,2(-0,67 ; 0,10)$ & 0,148 \\
\hline Vitamina D $(\mu g)$ & $-0,12$ & $0,1(-0,43 ; 0,19)$ & 0,448 & $-0,15$ & $7,9(-0,42 ; 0,11)$ & 0,257 \\
\hline Vitamina E (mg) & $-0,06$ & $0,1(-0,31 ; 0,43)$ & 0,756 & $-0,05$ & $7,9(-0,40 ; 0,31)$ & 0,798 \\
\hline \multicolumn{7}{|l|}{$\mathrm{CC}(\mathrm{cm})$} \\
\hline Cálcio (mg) & $-1,90$ & $2,3(-2,72 ;-1,08)$ & $<0,001$ & $-1,26$ & $23,4(-1,94 ;-0,59)$ & $<0,001$ \\
\hline Ferro (mg) & $-0,15$ & $0,0(-1,11 ; 0,81)$ & 0,757 & $-0,53$ & $22,7(-1,06 ;-0,01)$ & 0,046 \\
\hline Zinco (mg) & 0,21 & $0,0(-1,22 ; 1,64)$ & 0,771 & $-0,07$ & $22,5(-0,78 ; 0,64)$ & 0,838 \\
\hline Vitamina A ( $\mu g R E)$ & $-1,61$ & $1,7(-2,48 ;-0,75)$ & $<0,001$ & $-0,92$ & $23,0(-1,76 ;-0,08)$ & 0,032 \\
\hline Vitamina C (mg) & $-0,41$ & $0,1(-1,36 ; 0,53)$ & 0,384 & $-0,63$ & $22,7(-1,52 ; 0,26)$ & 0,164 \\
\hline Vitamina D $(\mu \mathrm{g})$ & $-0,60$ & $0,3(-1,38 ; 0,17)$ & 0,124 & $-0,69$ & $22,8(-1,32 ;-0,06)$ & 0,033 \\
\hline Vitamina E (mg) & 0,07 & $0,0(-0,75 ; 0,88)$ & 0,870 & $-0,07$ & $22,5(-0,76 ; 0,63)$ & 0,845 \\
\hline
\end{tabular}

ß: coeficiente de regressão; CC: circunferência da cintura; IC95\%: intervalo de 95\% de confiança; IMC: índice de massa corporal;

R2: coeficiente de determinação.

* A amostra analítica foi constituída por um $n=1.051$, uma vez que foram incluídos nas análises apenas os registros com dados completos para todas as variáveis;

** Análise ajustada para as variáveis sexo, idade, estado civil, cor da pele, escolaridade, renda familiar per capita;

*** Valor de p do teste de Wald.

A e vitamina D mostrou-se associado com maiores valores do IMC e da CC. Por outro lado, entre os indivíduos de 50 a 63 anos, verificou-se uma relação direta entre o consumo desses micronutrientes e valores elevados do IMC e da CC.

Conforme indicado na literatura 1,2, as taxas de obesidade dobraram mundialmente desde 1980, resultando em um contingente de mais de 600 milhões de adultos obesos. Nesse cenário, pesquisas publicadas nos últimos cinco anos têm associado o aumento da doença, entre outros fatores, ao consumo insuficiente de micronutrientes essenciais 10,33. Isso ocorre porque, nas sociedades ocidentais, o aumento da ingestão de calorias totais decorrente do processo de transição nutricional tem sido associado ao aumento progressivo do consumo de produtos ultraprocessados 34,35,36. Como resultado, estudos no Brasil, México, Estados Unidos, Reino Unido e Alemanha têm mostrado que pelo menos metade da população adulta nesses países não atinge a quantidade mínima recomendada de consumo de um número considerável de vitaminas e minerais, além de quantificar prevalências elevadas de obesidade (geral e abdominal) 14,15,34,36.

Postula-se, nesse cenário, que tanto o consumo insuficiente quanto o metabolismo prejudicado de absorção dos micronutrientes no indivíduo obeso possam estar envolvidos com o ganho de peso e/ou manutenção da obesidade 33. Um exemplo disso é o que ocorre com o ferro. Segundo uma metanálise publicada em 2015, além de o baixo consumo do micronutriente favorecer a obesidade, no indivíduo obeso, a própria inflamação que acompanha a obesidade pode diminuir a sua absorção e contribuir para a manutenção e/ou exacerbação desse agravo 37.

No presente estudo, em conformidade com tais achados, foi encontrada uma associação entre o menor consumo de ferro e valores elevados do IMC e da CC. Esses resultados assemelham-se aos de um estudo transversal com amostra representativa de mulheres do México, que também identificou 


\section{Figura 2}

Associação * entre o consumo alimentar de micronutrientes (cálcio, vitamina A, vitamina D) ** e os indicadores de estado nutricional (índice de massa corporal - IMC - e circunferência da cintura - CC), estratificada pela idade. Estudo EpiFloripa Adultos 2012, Florianópolis, Santa Catarina, Brasil, 2012.

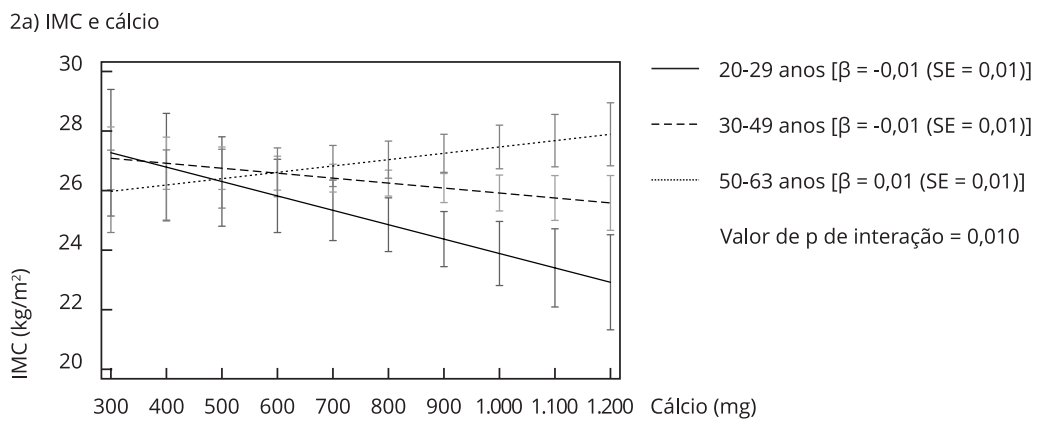

2b) IMC e vitamina A

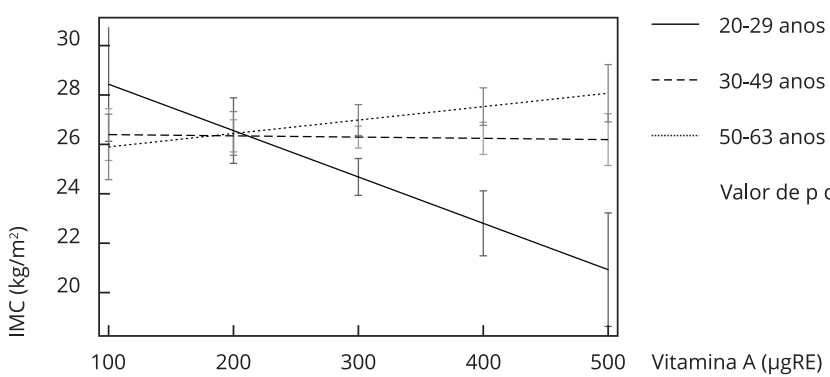

2c) IMC e vitamina D

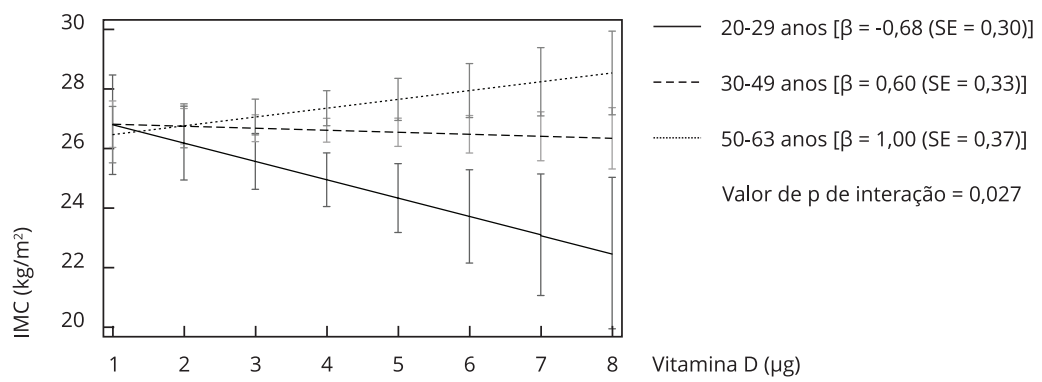

2d) CC e cálcio

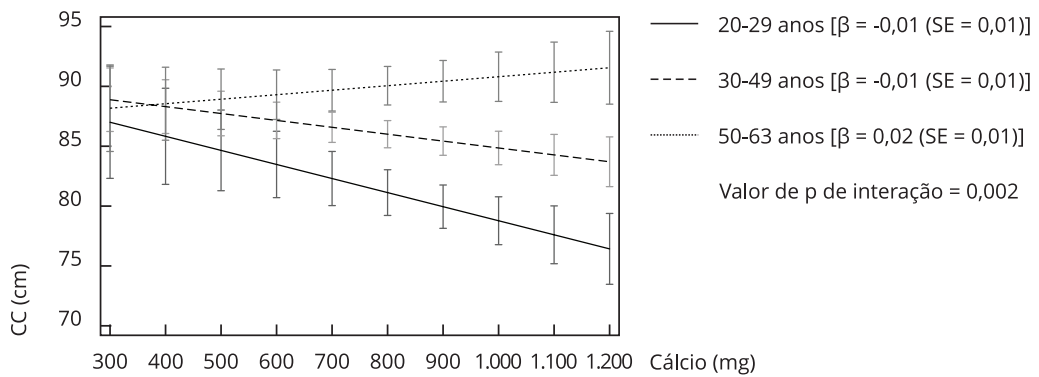

(continua) 
2e) CC e vitamina A

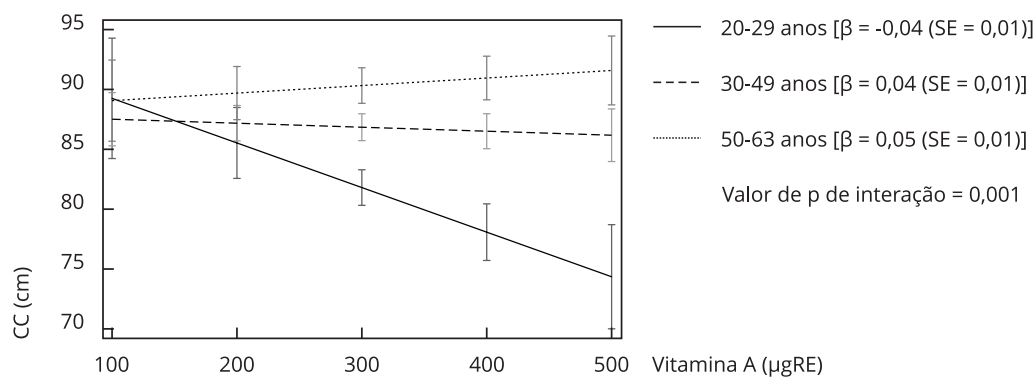

2f) CC e vitamina $D$

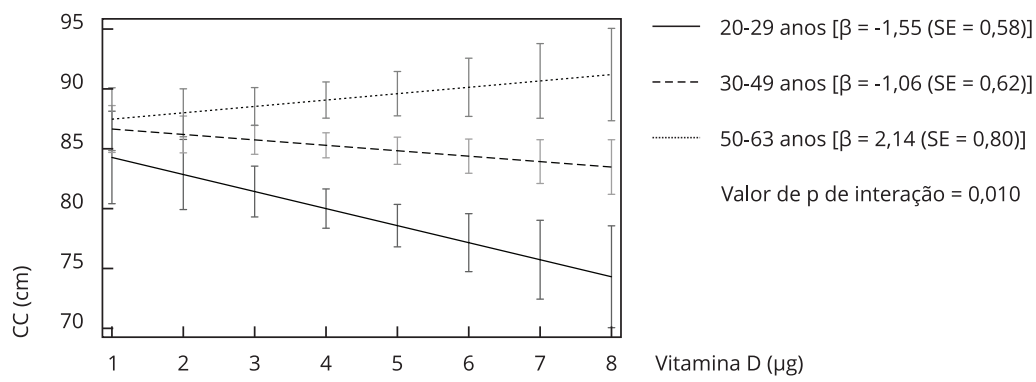

$\beta$ : coeficiente de regressão; SE: erro padrão.

* Análises ajustadas para as variáveis sexo, idade, estado civil, cor da pele, escolaridade, renda familiar per capita;

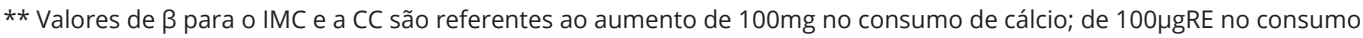
de vitamina $A$, e de $1 \mu$ g no consumo da vitamina $D$.

uma correlação negativa entre o consumo insuficiente do micronutriente e valores elevados do IMC $\left(\geq 30 \mathrm{~kg} / \mathrm{m}^{2}\right.$ ), além de uma probabilidade de obesidade geral quase duas vezes maior entre mulheres com deficiência de ferro 11. A gravidade desses resultados concentra-se sobretudo no fato de que, além de favorecer a obesidade, o consumo inadequado de ferro ainda contribui para a ocorrência da anemia, doença com importantes repercussões negativas à saúde. 11,37.

Outro micronutriente neste estudo que também mostrou associação com valores elevados do IMC e da CC em adultos jovens foi o cálcio. Tais achados se assemelham aos resultados de outros estudos transversais na literatura, como o The Israeli National Health and Nutrition Study em Israel 8, o The Quebec Family Study no Canadá 38 e o Encuesta Nacional de Salud y Nutrición no México 12. Em todos esses estudos, também foi identificada associação inversa entre o consumo inadequado do micronutriente e valores do IMC e/ou da CC elevados.

Valores elevados da CC em adultos jovens também se mostraram associados com o consumo insuficiente das vitaminas $\mathrm{A}$ e $\mathrm{D}$, resultados que se assemelham aos de pesquisas em países de renda média 39 e alta 9. No México, Garcia et al. 39 identificaram, após análise de dados transversais, que mulheres com obesidade abdominal apresentavam consumo de vitamina A mais inadequado quando comparadas com mulheres sem obesidade. Nos Estados Unidos, Maki et al. 9, analisando dados longitudinais, encontraram associação inversa entre o consumo insuficiente de vitamina $\mathrm{D}$ e valores elevados da CC em adultos.

A preocupação com esse cenário é que, além de o consumo insuficiente desses micronutrientes mostrar associação com o estado nutricional de obesidade, investigações acerca do tema têm cha- 


\section{Figura 3}

Associação * entre o consumo alimentar de micronutrientes (ferro e zinco) ** e a circunferência da cintura (CC), estratificada pela idade. Estudo EpiFloripa Adultos 2012, Florianópolis, Santa Catarina, Brasil, 2012.

3a) CC e ferro

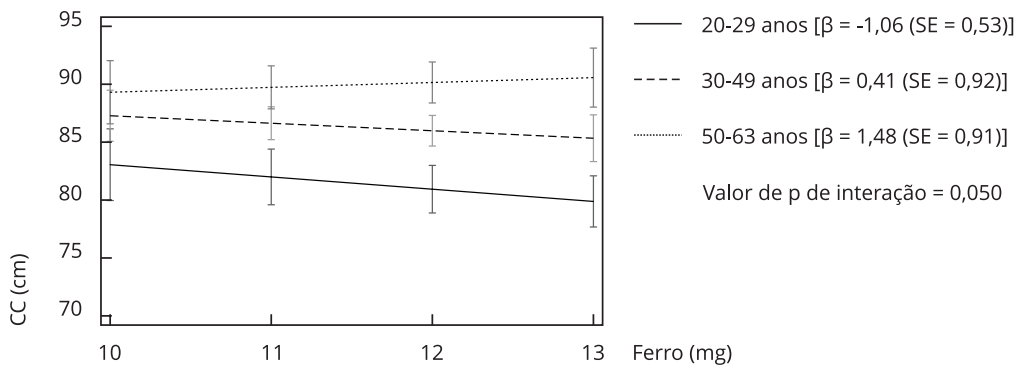

3b) CC e zinco

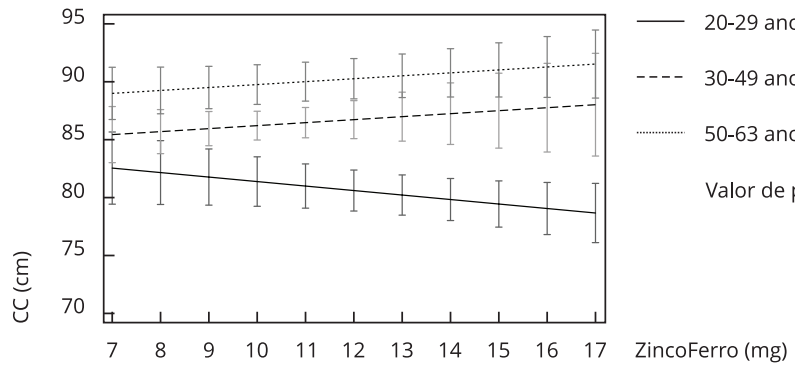

$\beta$ : coeficiente de regressão; SE: erro padrão.

* Análises ajustadas para as variáveis sexo, idade, estado civil, cor da pele, escolaridade, renda familiar per capita;

* Valore de $\beta$ para a circunferência da cintura são referentes ao aumento de $1 \mathrm{mg}$ no consumo de ferro e de

$1 \mathrm{mg}$ no consumo de zinco.

mado a atenção também para uma maior probabilidade de outras desordens metabólicas associadas com ambas as condições 12,33,40,41. Por exemplo, o consumo insuficiente de cálcio ao promover a deposição de ácidos graxos nos adipócitos tem sido associado não só à obesidade mas também a uma maior probabilidade de problemas cardiovasculares 12,33,38. A deficiência de vitamina D, por sua vez, ao exercer impacto importante sobre a deposição de gordura na região abdominal, tem sido associada a maior probabilidade de diabetes e hipertensão 9,33,40. Já a deficiência de vitamina A, ao promover a elevação da proteína ligadora de retinol, tem sido correlacionada com a obesidade abdominal, mas também com resistência a insulina, dislipidemia e hipertensão 33,41. A gravidade desses achados está no fato de que tais doenças crônicas não transmissíveis (DCNT) repercutem sobre as taxas de morbimortalidade em todo o mundo. Só no Brasil, estima-se que essas DCNT juntas respondam por quase $72 \%$ dos óbitos no país 6,42.

Considerando-se que, na amostra avaliada, a análise do IMC não refletiu deficiência energética, uma vez que somente $1,2 \%$ da amostra apresentou déficit de peso, tudo levar a crer que as associações encontradas sejam decorrentes de baixo consumo dos micronutrientes investigados, e não à ingestão insuficiente de alimentos. Isso remete-se ao processo de transição nutricional verificado nas sociedades ocidentais. No Brasil, dados provenientes de estudos representativos do cenário nacional revelam como elementos negativos desse processo o aumento do consumo de refeições prontas, embutidos e bebidas açucaradas (produtos ultraprocessados), paralelamente à diminuição do consumo de legumi- 
nosas, cereais, frutas e vegetais 35,36,43. Como resultado, em menos de quatro décadas (1974 a 2009), houve não somente aumento da densidade energética das refeições, mas perda da qualidade nutricional da dieta, implicando nesse antagonismo de situações nutricionais no país obesidade e deficiências de micronutrientes $14,35,36,43$.

O entendimento desse cenário se faz fundamental, sobretudo porque contribui para a compreensão de outro resultado encontrado por este estudo: a heterogeneidade promovida pela idade nas associações entre o consumo alimentar de micronutrientes e os dois indicadores de estado nutricional. Conforme supracitado, entre indivíduos na faixa etária de 20 a 29 anos, o menor consumo de micronutrientes (cálcio, ferro, zinco, vitaminas A e D) esteve relacionado a maiores valores do IMC e/ou da CC. Em uma análise mais ampla e relacionada com o processo de transição nutricional no Brasil, esses indivíduos jovens são os que nasceram entre as décadas de 1980 e 1990, justamente o período da transição nutricional no país marcado pela redução da desnutrição e o aumento da obesidade. Isso significa que tais indivíduos viveram o seu desenvolvimento fetal e os primeiros anos de vida nesse período de mudanças.

Segundo Leon 44, durante a gestação e os dois primeiros anos de vida, o aporte insuficiente de micronutrientes acompanhado do excesso de macronutrientes sobretudo após esse período predispõe os indivíduos expostos a tal condição a consequências adversas à saúde na vida adulta, como, por exemplo, à obesidade. Isso se dá porque, durante esses estágios da vida, dietas com elevada densidade energética e limitadas em micronutrientes repercutem em aumento do tecido adiposo em lugar de tecido muscular e massa óssea. Provavelmente seja decorrente desses achados a maior predisposição observada à obesidade neste estudo entre indivíduos de 20 a 29 anos, sobretudo porque os hábitos alimentares formados na infância tendem a persistir na idade adulta.

Embora esse papel das deficiências de micronutrientes como determinantes precoces da obesidade no adulto não esteja bem esclarecido entre populações de países de renda média, essa teoria do desenvolvimento precoce de doenças pode talvez ajudar a elucidar a heterogeneidade dos resultados deste estudo relacionada com a idade. Afinal, deve-se considerar que, entre os participantes de 50 a 63 anos, a mesma tendência não se repetiu, o que pode estar relacionado ao fato de esses participantes representarem os sobreviventes em seu grupo etário, tendo em vista que o envelhecimento associado à obesidade tende a contribuir para uma maior carga de DCNT e mortalidade. Exemplo disso são as evidências na literatura que mostram maior probabilidade de doenças cardiovasculares entre adultos mais velhos com obesidade e consumo inadequado de micronutrientes como cálcio e/ou vitamina D 13,40,45.

Em face disso, assegurar uma dieta de qualidade nutricional durante o desenvolvimento intrauterino, bem como durante o período pós-natal, pode ser um caminho factível para quebrar o ciclo intergeracional da obesidade e de outras DCNT do adulto. Além disso, é consensual que níveis dietéticos adequados de vitaminas (A, C, D e E) e minerais (cálcio, ferro e zinco) ao longo da vida são imprescindíveis para a boa saúde, cursando com menores taxas de mortalidade, hospitalizações e incapacidades por DCNT 33.

Como limitações do presente estudo, destacamos o uso do R24h para a obtenção dos dados do consumo alimentar. É possível que a aplicação do instrumento em apenas dois momentos (um no total da amostra e outro em subamostra dos respondentes) possa não ter contemplado toda a variabilidade do consumo alimentar habitual. Porém, tal metodologia foi adotada considerando os achados da literatura 21. Segundo Verly-Junior et al. 21, taxas de reaplicação do R24h acima de 40\% garantem que não haja perda importante de precisão na avaliação dietética de nutrientes. Além disso, considerando que o R24h depende da memória do entrevistado, todos os R24h foram administrados por entrevistadores treinados e seguindo o Multiple Pass Method 22, minimizando, assim, a possibilidade desse viés. Outra limitação deste estudo refere-se ao uso do NDSR, uma vez que esse software apresenta como base de dados principal uma tabela norte-americana, e não brasileira. Visando a contornar tal limitação, diversos procedimentos metodológicos foram adotados, conforme descrito na seção de métodos. Adicionalmente, o delineamento transversal também se perfaz em mais uma limitação do estudo, tendo em vista a impossibilidade de se estabelecer temporalidade nas associações testadas (causalidade reversa). Por fim, ressaltamos que, apesar de o presente estudo ter feito uma análise da inadequação da vitamina D na perspectiva do consumo alimentar, a principal fonte dessa vitamina não é dietética, mas a exposição solar. Conforme a literatura, uma exposição solar moderada (duas a três vezes por semana) já se mostraria suficiente para satisfazer as necessidades orgânicas desse 
micronutriente, independentemente da ingestão alimentar 14,31. Além disso, é preciso considerar que os valores da EAR para a vitamina $\mathrm{D}$ foram estabelecidos com base na exposição solar da população norte-americana 31 , motivo pelo qual a prevalência de inadequação do micronutriente encontrada por este estudo provavelmente esteja superestimada.

Em conclusão, os resultados desta pesquisa mostraram claramente associação inversa entre o menor consumo de cálcio e ferro e maiores valores do IMC e da CC, e entre o menor consumo das vitaminas A e D e maiores valores da CC, além de um padrão distinto nessas associações conforme a idade. Assim, da perspectiva da saúde pública, espera-se que esses resultados possam contribuir para um maior conhecimento acerca do tema investigado, além de fortalecer as ações de âmbito coletivo voltadas para promover hábitos alimentares saudáveis na população. Desse modo, almeja-se não apenas que as deficiências no consumo de micronutrientes sejam reduzidas, mas também as prevalências da obesidade e de outras doenças crônicas subjacentes a ambas as condições. Ressalta-se, por fim, que não é recomendável que o aumento da ingestão de vitaminas e minerais se baseie na suplementação e/ou na fortificação de alimentos, considerando que tais estratégias podem desestimular a adoção de hábitos alimentares saudáveis pela população 14,46 .

\section{Colaboradores}

Todos os autores participaram igualmente na concepção do trabalho, análise e interpretação dos dados, redação do artigo e aprovação da versão final submetida. Também são responsáveis por todos os aspectos do trabalho, na garantia da exatidão e integridade de qualquer parte da obra.

\section{Agradecimentos}

Agradecemos aos participantes do estudo, à Coordenação de Aperfeiçoamento de Pessoal de Nível Superior (Capes) pela bolsa de doutorado concedida a F. Cembranel, e ao Conselho Nacional de Desenvolvimento Científico e Tecnológico (CNPq) pelo financiamento do projeto EpiFloripa Adultos, nas ondas 2009 e 2012 - Estudo Epidemiológico das Condições de Saúde dos Adultos do Município de Florianópolis, Santa Catarina, Brasil (editais universais no 485327/2007-4 e no 477061/2010-9), do qual o presente artigo faz parte. 


\section{Referências}

1. World Health Organization. Obesity and overweight. http://www.who.int/mediacentre/ factsheets/fs311/en/ (acessado em Ago/2016)

2. NCD Risk Factor Collaboration. Trends in adult body-mass index in 200 countries from 1975 to 2014: a pooled analysis of 1698 population-based measurement studies with 19.2 million participants. Lancet 2016; 387:137796.

3. Li C, Ford ES, McGuire LC, Mokdad AH. Increasing trends in waist circumference and abdominal obesity among U.S. adults. Obesity 2007; 15:216-24

4. Howel D. Trends in the prevalence of abdominal obesity and overweight in English adults (1993-2008). Obesity 2012; 20:1750-2.

5. Instituto Brasileiro de Geografia e Estatística. Pesquisa Nacional de Saúde 2013: percepção do estado de saúde, estilos de vida e doenças crônicas no Brasil, nas grandes regiões e unidades da Federação. Rio de Janeiro: Instituto Brasileiro de Geografia e Estatística; 2015.

6. Djalalinia S, Qorbani M, Peykari N, Kelishadi R. Health impacts of obesity. Pak J Med Sci 2015; 31:239-42.

7. Popkin BM, Adair LS, Ng SW. Global nutrition transition and the pandemic of obesity in developing countries. Nutr Rev 2012; 70:3-21.

8. Dicker D, Belnic Y, Goldsmith R, Kaluski DN. Relationship between dietary calcium intake, body mass index, and waist circumference in MABAT: The Israeli National Health and $\mathrm{Nu}-$ trition Study. Isr Med Assoc J 2008; 10:512-5.

9. Maki KC, Fulgoni VL, Keast DR, Rains TM, Park KM, Rubin MR. Vitamin D intake and status are associated with lower prevalence of metabolic syndrome in U.S. Adults: National Health and Nutrition Examination Surveys 2003-2006. Metab Syndr Relat Disord 2012; 10:363-72.

10. Agarwal S, Reider C, Brooks JR, Fulgoni VL. Comparison of prevalence of inadequate nutrient intake based on body weight status of adults in the United States: an analysis of NHANES 2001-2008. J Am Coll Nutr 2015; 34:126-34.

11. Cepeda-Lopez AC, Osendarp SJM, MelseBoonstra A. Sharply higher rates of iron deficiency in obese Mexican women and children are predicted by obesity-related inflammation rather than by differences in dietary iron intake. Am J Clin Nutr 2011; 93:975-83.

12. González AM, Campos-Nonato IR, Hernández-Barrera L, Flores-Aldana ME. Asociación entre la ingesta de calcio dietético y el índice de masa corporal elevado en adultos mexicanos de 20 a 59 años de edad: estudio de corte transversal. Medwave 2013; 13:5635.

13. Pinheiro MM, Ciconelli RM, Villaça-Chaves G, Aquino L, Juzwiak CR, Genaro PS, et al. Antioxidant intake among Brazilian adults - The Brazilian Osteoporosis Study (BRAZOS): a cross-sectional study. Nutr J 2011; 10:39.
14. Araujo MC, Bezerra IN, Barbosa FS, Junger WL, Yokoo EM, Pereira RA, et al. Consumo de macronutrientes e ingestão inadequada de micronutrientes em adultos. Rev Saúde Pública 2013; 47 Suppl 1:177S-89S.

15. Barquera S, Hernández-Barrera L, CamposNonato I, Espinosa J, Flores M, Barriguete $\mathrm{JA}$, et al. Energy and nutrient consumption in adults: analysis of the Mexican National Health and Nutrition Survey 2006. Salud Pública Méx 2009; 51:562-73.

16. Boing AC, Peres KG, Boing AF, Hallal PC, Silva NN, Peres MA. EpiFloripa Health Survey: the methodological and operational aspects behind the scenes. Rev Bras Epidemiol 2014; 17:147-62.

17. World Health Organization. Physical status: the use and interpretation of anthropometry. Geneva: World Health Organization; 1995. (WHO Technical Report Series, 854).

18. Lohman TG, Roche AF, Martorell R. Anthropometric standardization reference manual. Champaign: Human Kinetics Books; 1988.

19. World Health Organization. Waist circumference and waist-hip ratio. Geneva: World Health Organization; 2008.

20. Willett WC, Howe GR, Kushi LH. Adjustment for total energy intake in epidemiologic studies. Am J Clin Nutr 1997; 65(4 Suppl):1220S$8 \mathrm{~S}$.

21. Verly Jr. E, Castro MA, Fisberg RM, Marchioni DM. Precision of usual food intake estimates according to the percentage of individuals with a second dietary measurement. J Acad Nutr Diet 2012; 112:1015-20.

22. Conway JM, Ingwersen LA, Moshfegh AJ. Accuracy of dietary recall using the USDA fivestep multiple-pass method in men: An observational validation study. J Am Diet Assoc 2004; 104:595-603.

23. Fisberg RM, Marchioni DML. Manual de avaliação do consumo alimentar em estudos populacionais: a experiência do inquérito de saúde em São Paulo (ISA). São Paulo: Faculdade de Saúde Pública, Universidade de São Paulo; 2012.

24. Pinheiro ABV, Lacerda EMA, Benzecry EH, Gomes MCS, Costa VM. Tabela para avaliação de consumo alimentar em medidas caseiras. 5a Ed. Rio de Janeiro: Editora Atheneu; 2010.

25. Núcleo de Estudos e Pesquisas em Alimentação, Universidade Estadual de Campinas. Tabela brasileira de composição de alimentos (TACO). 4a Ed. Campinas: Núcleo de Estudos e Pesquisas em Alimentação, Universidade Estadual de Campinas; 2011.

26. Institute of Medicine. Dietary reference intakes: the essential guide to nutrient requirements. Washington DC: National Academy Press; 2006. 
27. Dodd KW, Guenther PM, Freedman LS, Subar AF, Kipnis V, Midthune D, et al. Statistical methods for estimating usual intake of nutrients and foods: a review of the theory. J Am Diet Assoc 2006; 106:1640-50.

28. Willett WC. Nutritional epidemiology. 3rd Ed. Oxford: Oxford University Press; 2013.

29. Institute of Medicine. Dietary reference intakes for vitamin C, vitamin E, selenium, and carotenoids. Washington DC: National Academy Press; 2000.

30. Institute of Medicine. Dietary reference intakes for vitamin $\mathrm{A}$, vitamin $\mathrm{K}$, arsenic, boron, chromium, copper, iodine, iron, manganese, molybdenum, nickel, silicon, vanadium and zinc. Washington DC: National Academy Press; 2001.

31. Institute of Medicine. Dietary reference intakes for calcium and vitamin D. Washington DC: National Academy Press; 2011.

32. Rothman KJ, Greenland S. Epidemiologia moderna. 3a Ed. São Paulo: Editora Artmed; 2011.

33. Troesch B, Biesalski HK, Bos R, Buskens E, Calder PC, Saris WHM, et al. Increased intake of foods with high nutrient density can help to break the intergenerational cycle of malnutrition and obesity. Nutrients 2015; 7:6016-37.

34. roesch B, Hoeft B, McBurney M, Eggersdorfer $\mathrm{M}$, Weber P. Dietary surveys indicate vitamin intakes below recommendations are common in representative Western countries. Br J Nutr 2012; 108:692-8.

35. Louzada MLC, Martins APB, Canella DS, Baraldi LG, Levy RB, Claro RM, et al. Impact of ultra-processed foods on micronutrient content in the Brazilian diet. Rev Saúde Pública $2015 ; 49: 45$.

36. Canella DS, Levy RB, Martins APB, Claro RM, Moubarac JC, Baraldi LG, et al. Ultraprocessed food products and obesity in Brazilian households (2008-2009). PLoS One 2014; 9:e92752.

37. Zhao L, Zhang X, Shen Y, Fang X, Wang Y, Wang F. Obesity and iron deficiency: a quantitative metaanalysis. Obes Rev 2015; 16:108193.
38. Jacqmain M, Doucet E, Després JP, Bouchard C, Tremblay A. Calcium intake, body composition, and lipoprotein-lipid concentration in adults. Am J Clin Nutr 2003; 77:1448-52.

39. Garcia OP, Ronquillo D, Caamaño MC, Camacho M, Long KZ, Rosado J. Zinc, vitamin A, and vitamin $C$ status are associated with leptin concentrations and obesity in Mexican women: results from a cross-sectional study. Nutr Metab (Lond) 2012; 9:59.

40. Cheng S, Massaro JM, Fox CS, Larson MG, Keyes MJ, McCabe EL, et al. Adiposity, cardiometabolic risk and vitamin D status: the Framingham Heart Study. Diabetes 2010; 59:242-8.

41. Kloting N, Graham TE, Berndt J, Kralisch S, Kovacs P, Wason CJ, et al. Serum retinol-binding protein is more highly expressed in visceral than in subcutaneous adipose tissue and is a marker of intra-abdominal fat mass. Cell Metab 2007; 6:79-87.

42. Schmidt MI, Duncan BB, Silva GA, Menezes AM, Monteiro CA, Barreto SM, et al. Chronic non-communicable diseases in Brazil: Burden and current challenges. Lancet 2011; 377:1949-61.

43. Instituto Brasileiro de Geografia e Estatística. Pesquisa de Orçamentos Familiares 20082009: antropometria e estado nutricional de crianças, adolescentes e adultos no Brasil. Rio de Janeiro: Instituto Brasileiro de Geografia e Estatística; 2010.

44. Leon DA. Biological theories, evidence, and epidemiology. Int J Epidemiol 2004; 33:116771.

45. Carvalho LS, Sposito AC. Vitamin D for the prevention of cardiovascular disease: are we ready for that? Atherosclerosis 2015; 241:72940.

46. Cembranel F, González-Chica DA, d’Orsi E. Inadequações na ingestão dietética de micronutrientes por homens e mulheres residentes no Sul do Brasil: Estudo EpiFloripa Adultos 2012. Cad Saúde Pública 2016; 32:e00164015. 


\section{Abstract}

The study's objective was to investigate the association between inadequate dietary consumption of micronutrients and indicators of general and abdominal obesity. Cross-sectional analysis of the second wave of the EpiFloripa Adults longitudinal study, including 1,222 individuals, aged 22-63 years and residing in Florianópolis, Santa Catarina State, Southern Brazil. Food consumption data was obtained from two 24-hour food recalls, and habitual consumption and prevalence rates of inadequate consumption of calcium, iron, zinc, and vitamins $A, C, D$, and $E$ were estimated according to the Institute of Medicine and National Research Council guidelines. General obesity was defined based on the body mass index (BMI) values and abdominal obesity was based on waist circumference (WC) values. For most of the micronutrients investigated (calcium and vitamins $A, C, D$, and $E)$, consumption levels were below the recommendations, with high prevalence of inadequate consumption in the sample as a whole. Only inadequate vitamin $C$ intake was higher among obese individuals (general or abdominal). In addition, there was an inverse association between lower consumption of calcium and iron and higher BMI and WC, and between lower consumption of vitamins $A$ and $D$ and higher WC levels $(\beta=-0.92 \mathrm{~cm}$; 95\%CI: $-1.76 ;-0.08$ and $\beta=-0.69 \mathrm{~cm} ; 95 \% \mathrm{CI}$ : - 1.32; -0.06, respectively), especially in young adults. The study showed an inverse association between inadequate dietary consumption of micronutrients and general and abdominal obesity in a sample of adults in Southern Brazil.

Food Consumption; Micronutrients; Obesity

\section{Resumen}

El El objetivo del estudio fue investigar la asociación entre el consumo alimentario inadecuado de micronutrientes e indicadores de obesidad general y abdominal. Métodos: análisis transversal, de la segunda fase del estudio longitudinal EpiFloripa Adultos, incluyendo 1.222 individuos de 22-63 años, residentes en Florianópolis, Santa Catarina, Sur de Brasil. Los datos de consumo alimentario fueron obtenidos mediante aplicación de dos recordatorios de 24 horas, siendo estimados, conforme las recomendaciones del Instituto de Medicina $y$ del Consejo Nacional de Investigación, de los EEUU. El estado nutricional de obesidad general fue determinado considerándose valores del indice de masa corporal (IMC), y la obesidad abdominal, considerándose valores de la circunferencia de la cintura (CC). Para la mayoría de los micronutrientes investigados (calcio, vitaminas $A, C, D$ $y E$ ), las medias de consumo fueron menores que las recomendaciones, con prevalencias elevadas de inadecuación para toda la muestra. Solamente la inadecuación en el consumo de vitamina $C$ fue mayor entre individuos obesos (general o abdominal). Además, asociación inversa fue observada entre el menor consumo de calcio e hierro con valores mayores de IMC y de la CC, y entre el menor consumo de vitaminas $A$ y $D$ con valores mayores de la CC ( $\beta=-0,92 \mathrm{~cm}$; IC95\%: $-1,76 ;-0,08$ y $\beta=$ -0,69cm; IC95\%: - 1,32; -0,06), principalmente entre adultos jóvenes. Fue posible identificar la existencia de asociación inversa entre el consumo alimentario inadecuado de micronutrientes y el estado nutricional de obesidad general y abdominal en una muestra de adultos residentes en el Sur de Brasil.

Consumo de Alimentos; Micronutrientes;

Obesidad
Recebido em 02/Ago/2016

Versão final reapresentada em 15/Jan/2017

Aprovado em 06/Fev/2017 